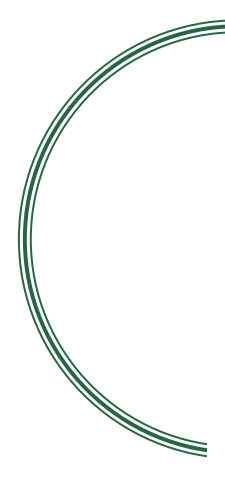

\title{
Oxidative Stress in Biology and Medicine
}

\author{
Daniel Tsun-Yee Chiu \\ Professor of Chang Gung University, Taiwan \\ Associate Editor of Biomedical Journal \\ President of SFRR-Asia (2014-2015)
}

$\mathrm{I}_{\mathrm{i}}^{\mathrm{n}}$

$\mathrm{n}$ this issue of Biomedical Journal, we have organized a special section related to "oxidative stress

in biology and medicine" with four invited review articles. These four articles are written by invited speakers of the "Biennial Meeting of Society for Free Radical Research-Asia" held in Chang Gung University of Taiwan from October 16 to 19, 2013. The first two review articles in this special section are written by Professor Barry Halliwell of Singapore and Professor Etsuo Niki of Japan, respectively, and are part of their Plenary Lecture during the aforementioned meeting. The third review article is written by Professor Steven Qian and his associate of the United States. The fourth review article is written by Professor Jiangang Shen and his associates of Hong Kong.

Professor Barry Halliwell's review article is entitled "Cell culture, oxidative stress, and antioxidants: Avoiding pitfalls." ${ }^{[1]}$ In this article, Professor Halliwell points out the potential artifacts created by reagents, particularly "antioxidants," because these chemicals can react with the constituents of the cell culture media to produce $\mathrm{H}_{2} \mathrm{O}_{2}$ and degradation products which can influence cell behavior. This article would be extremely helpful to the free radical/antioxidant community to have greater awareness of the potential artifacts in cell culture studies.

The second article written by Professor Etsuo Niki is entitled "Antioxidants: Basic principles, emerging concepts, and problems." ${ }^{[2]}$ It is well accepted that radical-scavenging antioxidants play an essential role in the maintenance of health and prevention of diseases. However, Professor Niki points out in his article that there is no evidence to support the use of non-discriminative antioxidant supplement for prevention of diseases. On the other hand, recent data show that antioxidants may be effective in the prevention and/or treatment of diseases when the right antioxidant is given to the right subject at the right time for the right duration. Recent findings in a clinical study by Professor Chandan Sen and his associates that tocotrienol provides multimodal protection against stroke (Oxygen Club of California Meeting at Davis, CA, USA, 2014) provide additional strong support to this concept. ${ }^{[3]}$

The third article written by Professor Steven Qian and his associate is entitled "Anti-cancer activities of $\omega-6$ polyunsaturated fatty acids." ${ }^{[4]}$ Although it is well-known that the $\omega-6$ s are abundant in our daily diet and could be implicated in many pathological processes including cancer development, the beneficial effects of $\gamma$-linolenic acid (GLA) and dihomo- $\gamma$-linolenic acid (DGLA), two of arachidonic acid upstream $\omega-6 \mathrm{~s}$, have been just started to be recognized. In this paper, Professor Qian reviews the documented anticancer activities of $\omega-6$ polyunsaturated fatty acids (PUFAs), including the recent findings regarding the anticancer effects of free radical-mediated DGLA peroxidation. Such an understanding of the beneficial effects of $\omega-6$ PUFAs would be helpful for the development of a $\omega-6-$ based diet care strategy for cancer prevention and treatment.

The fourth article written by Professor Jiangang Shen and his associates is entitled "Pros and cons of current approaches for detecting peroxynitrite and their applications." ${ }^{\text {"[] }}$ Peroxynitrite, a representative of reactive nitrogen species, plays important roles in the physiological and pathological processes of health and diseases. However, due to its high reactivity and short half-life, accurate measurement of peroxynitrite in biological systems is a great challenge. In this article, three major approaches for peroxynitrite detec- 
tion, including electrochemical sensors, detection of nitrotyrosine formation, and fluorescent probes, are reviewed. These techniques would be helpful for the further delineation of the roles of peroxynitrite in the oxidative stress related physiological and pathological conditions and provide platforms for drug discovery targeting peroxynitrite for therapeutic purposes.

All in all, these four review articles are a feast for readers interested in free radicals in biology and medicine. These articles provide cutting-edge knowledge in redox-related biological issues including pitfalls in tissue culture, new concepts in antioxidant supplementation, anticancer effects of $\omega-6$ PUFAs, and methods used in detecting peroxynitrite. We are sure that this special section would satisfy the appetite of the readers craving for knowledge in redox biology and medicine.

\section{REFERENCES}

1. Haliwell B. Cell culture, oxidative stress, and antioxidants: Avoiding pitfalls. Biomed J 2014;37:99-105.

2. Niki E. Antioxidants: Basic principles, emerging concepts, and problems. Biomed J 2014;37:106-11.

3. Sen CK. $\alpha$-Tocotrienol: Multimodal protection against stroke. Oxygen Club of California World Congress: 7-10 May 2014; Davis. UC Davis; 2014. p. 42.

4. Qian SY. Anti-cancer activities of $\omega-6$ polyunsaturated fatty acids. Biomed J 2014;37:112-9.

5. Chen X, Chen H, Deng R, Shen J. Pros and cons of current approaches for detecting peroxynitrite and their applications. Biomed J 2014;37:120-6. 